\title{
Postoperative emergence agitation and intraoperative sevoflurane sedation under caudal block in children: a randomized comparison of two sevoflurane doses
}

Received October 12, 2018

Revised 1st, November 13, 2018

2nd, November 20, 2018

3rd, December 3, 2018

4th, December 18, 2018

Accepted December 18, 2018

\author{
Corresponding author \\ Sungsik Park, M.D., Ph.D. \\ Department of Anesthesiology and \\ Pain Medicine, School of Medicine, \\ Kyungpook National University, \\ 130 Dongdeok-ro, Jung-gu, Daegu \\ 41944, Korea \\ Tel: 82-53-420-5877 \\ Fax: 82-53-426-2760 \\ E-mail: sspark@knu.ac.kr \\ ORCID \\ https://orcid.org/0000-0001-5574-7513
}

\section{Eun Kyung Choi', Suyong Park', Ki-bum Park², Kyung Hwa Kwak², and Sungsik Park ${ }^{3}$}

Department of Anesthesiology and Pain Medicine, ${ }^{1}$ Yeungnam University College of Medicine, ${ }^{2}$ Dongsan Hospital, Keimyung University School of Medicine, ${ }^{3}$ School of Medicine, Kyungpook National University, Daegu, Korea
Background: Sub-umbilical surgery under caudal block in conjunction with sevoflurane sedation may be safe in terms of maintaining spontaneous breathing and avoiding complications associated with general anesthesia. However, sevoflurane-induced emergence agitation (EA) continues to be a clinically important phenomenon in children. To compare the incidence of EA in children undergoing sub-umbilical surgery under caudal block with two different doses of sevoflurane.

Methods: Forty children (aged 1-5 years) scheduled to undergo inguinal hernia repair under caudal block with sevoflurane sedation via a face mask were randomized into either the low-dose (1.0\%) end-tidal sevoflurane concentration group (Group LS) or the high-dose (2.5\%) end-tidal sevoflurane concentration group (Group HS). We monitored EA episodes at 5 and $30 \mathrm{~min}$ in the post-anesthetic care unit (PACU) by using the fourpoint agitation scale and the Pediatric Anesthesia Emergence Delirium (PAED) scale.

Results: The four-point agitation scale scores and PAED scores were not different between the groups at 5 min. However, the agitation score was higher in Group HS than in Group LS at 30 min after arriving in the PACU. The time required to recover from sedation was longer in Group HS than in Group LS.

Conclusions: Face-mask sedation with 1.0\% sevoflurane in conjunction with caudal block may be more effective than that with $2.5 \%$ sevoflurane in preventing EA.

Keywords: Caudal extradural; Child; Emergence delirium; Sevoflurane.

\section{INTRODUCTION}

Caudal block is a common anesthetic option for pediatric patients undergoing sub-umbilical surgery. Most caudal blocks are performed as adjuvant procedures to general anesthesia with endotracheal intubation [1], but caudal block with sedation per se can be used as an alternative to general anesthesia [2-4]. Surgery under caudal block in conjunction with sedation may be safe in terms of maintaining spontaneous breathing and avoiding complications associated with general anesthesia. Specifically, caudal block under sedation has been considered effective in high-risk patients such as neonates and infants. Moreover, compared to general anesthesia, it has a lower likelihood of precipitating postoperative respiratory events [5].

Sevoflurane administration via a face mask is a popular

This is an Open Access article distributed under the terms of the Creative Commons Attribution Non-Commercial License (http://creativecommons.org/licenses/by-nc/4.0) which permits unrestricted non-commercial use, distribution, and reproduction in any medium, provided the original work is properly cited. 
anesthetic protocol for pediatric sedation, and this technique is commonly used in our institution. Despite various advantages of sevoflurane sedation, including rapid onset, quick recovery, reduced airway irritation, and greater hemodynamic stability, emergence agitation (EA) induced by sevoflurane is a major concern to pediatric anesthesiologists. EA is characterized by inconsolable crying, kicking, disorientation, and cognitive impairment, and can lead to significant problems such as the removal of drains or catheters, falling from the bed, and self-inflicted harm to the surgical wound $[6,7]$.

To date, no study has investigated EA caused by different sedation doses of sevoflurane in conjunction with caudal block. Therefore, we aimed to investigate whether sevoflurane sedation with caudal block may be an alternative anesthetic technique in children undergoing inguinal hernia repair and to compare the incidence of EA in children administered two different doses of sevoflurane.

\section{MATERIALS AND METHODS}

\section{Study design}

This study was approved by the Institutional Review Board (no. KNUH201606026), and informed consent was obtained from the parents of all the pediatric patients. The study was registered as a clinical trial (NCT03134547). Forty-three children, aged up to 5 years, scheduled to undergo elective sub-umbilical surgery were enrolled. Children with developmental delays, anxiety disorders, history of allergy to any study drugs, or severe systemic disease were excluded from this study. Randomization was performed with a computergenerated schedule by using a concealed random number table; each random number was sealed in an envelope. The children were randomized into either the low-dose (1.0\%) end-tidal sevoflurane concentration group (Group LS) or the high-dose (2.5\%) end-tidal sevoflurane concentration group (Group HS). After sedation with $2 \mathrm{mg} / \mathrm{kg}$ intravenous (IV) ketamine and $0.05 \mathrm{mg} / \mathrm{kg}$ IV midazolam during preoperative preparation, the patients were transferred to the operating room. Standardized monitoring (electrocardiography, pulse oximetry, and noninvasive blood pressure monitoring) was implemented, and capnography was performed via a face mask. Baseline vital signs were assessed with the patients in the supine position; thereafter, the patients were placed in the lateral Sims' position. With patients under 100\% oxygen and with sevoflurane administered via the face mask according to the study group protocol, $1 \mathrm{ml} / \mathrm{kg} 1 \%$ lidocaine with 5 $\mu \mathrm{g} / \mathrm{ml}$ epinephrine was administered into the caudal space by using a 22-gauge needle. During caudal block, $1 \mathrm{mg} / \mathrm{kg}$ IV ketamine was administered for ensuring patient immobility if needed. All procedures were performed by one skilled anesthesiologist who was blinded to the study group allocation. After caudal block was performed, the patients were returned to the supine position and maintained on spontaneous ventilation under $100 \%$ oxygen and sevoflurane administration via the face mask according to the study group protocol to achieve sedation during surgery. The face mask was tightly re-taped at the cheek to prevent sevoflurane leakage as much as possible. End-tidal concentrations of sevoflurane and carbon dioxide were continuously monitored during surgery. If the oxygen saturation dropped below $90 \%$, assisted ventilation was provided. The heart rate (HR) and mean blood pressure (MBP) were recorded at arrival, during the surgery (T0), just before caudal block (T1), just after caudal block (T2), 5 min after returning to the supine position (T3), 15 min after returning to the supine position (T4), 5 min before the end of the surgery (T5), 5 min after arrival in the post-anesthetic care unit (PACU) (T6), after $15 \mathrm{~min}$ in the PACU (T7), and after 30 min in the PACU (T8). If the patients moved intraoperatively, they were administered IV ketamine in $1 \mathrm{mg} / \mathrm{kg}$ increments. If the patients were receiving supplemental inotropic drugs or vasoconstrictors, the details were recorded (these drugs were administered when the HR and MBP were altered by more than $10 \%$ of their baseline values).

In the PACU, another anesthesiologist who was blinded to the study protocol monitored the incidence rate of EA (primary outcome) at T6 and T8. The EA scores (secondary outcome) at T6 and T8 were assessed using a four-point agitation scale (possible range, 1-4) [8] and the Pediatric Anesthesia Emergence Delirium (PAED) scale (possible range, 0-20) [6]. We also recorded the distribution of scores according to each EA scale [9]. A diagnosis of EA was considered when the fourpoint agitation scale score was $\geq 3$ or the PAED scale score was $\geq 10$ [9]. If the total score on the PAED scale was higher than 15 (described as severe agitation), $0.5 \mathrm{mg} / \mathrm{kg}$ propofol was administered to the patient via an injection. 


\section{Statistical analysis}

In this study, the primary outcome was the incidence rate of EA in the PACU. A power analysis to calculate the sample size was performed on the basis of our preliminary records, by comparing both concentrations of sevoflurane with the incidence of EA (four-point agitation scale score $\geq 3$, at 30 min after arrival in the PACU). A preliminary study on 20 patients undergoing inguinal hernia surgery under caudal block with low-dose (Group LS) and high-dose (Group HS) sevoflurane sedation showed that the incidence rate of EA in the PACU was 9.1\% (1/11) and 66.7\% (6/9), respectively. Considering the primary outcome of EA incidence rate in the two independent groups, this analysis revealed that we needed a sample size of 19 patients in each group to achieve $90 \%$ power and a $1 \%$ level of significance. Therefore, after accounting for a 5\% drop-out rate, we enrolled 20 patients in each group and a total of 40 patients into the study. The demographic data were summarized using descriptive analyses, with the quantitative variables being expressed as means \pm standard deviations and the qualitative variables being expressed as frequencies and percentages. The four-point agitation score and PAED score between Group LS and Group HS were compared using the chi-square test, and the results were expressed as frequencies, percentiles, and $\mathrm{P}$ values. To compare two population rates with independent samples, the rate comparison by two independent groups with $\mathrm{z}$ statistics was used, and the results were expressed as rate differences,

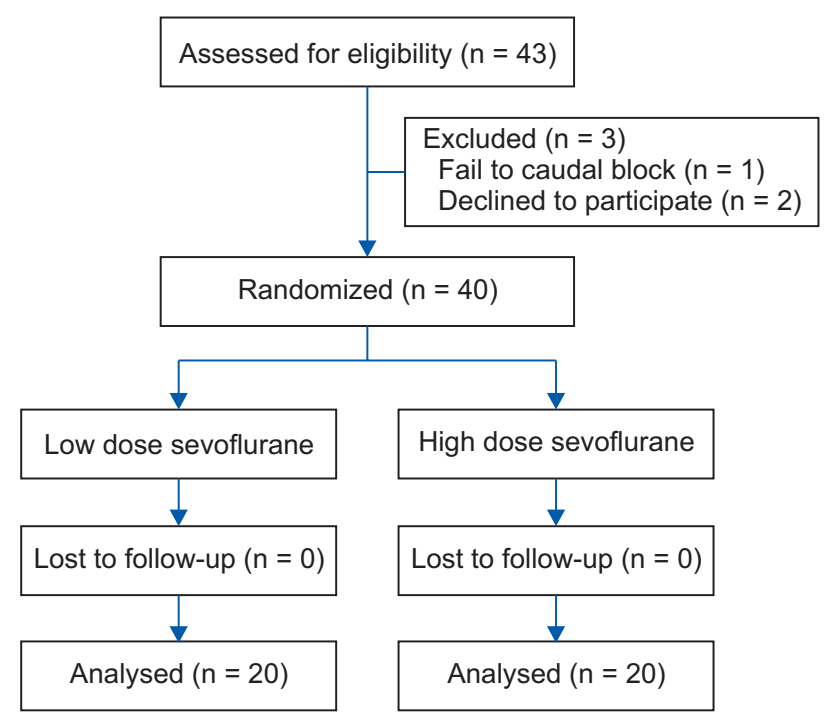

Fig. 1. Flow diagram of the study design and patient enrollment.
95\% confidence intervals (CIs) for rate differences, and P values. Comparison of the changes in hemodynamic parameters such as the HR and MBP caused by the time, group, and interaction (time difference by group) effects was performed using a generalized linear model. All data were analyzed by a medical statistician. Statistical analyses were performed using IBM SPSS statistics for Windows, version 23.0 (IBM Corp., USA). All the tests were two-sided, and P values less than 0.01 were considered statistically significant.

\section{RESULTS}

Forty-three children underwent the screening test; caudal block failed in one child, and two children declined to participate in the study. Finally, 40 children (20 in Group HS and 20 in Group LS) were enrolled and none of them dropped out (Fig. 1). No significant differences were observed in the age, sex, height, weight, duration of surgery or anesthesia, proportion of patients who required anesthetics during caudal block and surgery, and proportion of patients who required supplemental ventilation intraoperatively (Table 1). The distribution of the scores between Group LS and Group HS on the four-point agitation scale at $5 \mathrm{~min}$ after arrival in the PACU was not statistically significant $(\mathrm{P}=0.245)$; however,

Table 1. Demographic Data

\begin{tabular}{lcc}
\hline \multicolumn{1}{c}{ Variables } & $\begin{array}{c}\text { Group LS } \\
(\mathrm{n}=20)\end{array}$ & $\begin{array}{c}\text { Group HS } \\
(\mathrm{n}=20)\end{array}$ \\
\hline Age (mo) & $25.8 \pm 20.6$ & $34.5 \pm 24.6$ \\
Sex (M/F) & $17(85) / 3(15)$ & $15(75) / 5(25)$ \\
Height (cm) & $84.9 \pm 18.7$ & $91.9 \pm 17.6$ \\
Weight (kg) & $11.7 \pm 4.7$ & $13.3 \pm 5.0$ \\
Anesthetic time (min) & $67.5 \pm 28.4$ & $64.3 \pm 22.1$ \\
Operation time (min) & $48.5 \pm 26.8$ & $45.6 \pm 21.1$ \\
Awake time (min) & $2.6 \pm 2.1$ & $5.9 \pm 2.0$ \\
Movement during caudal block & $5(25)$ & $2(10)$ \\
$\quad$ Supplemental anesthetics & $5(25)$ & $2(10)$ \\
$\quad$ ketamine) & $0(0)$ & $0(0)$ \\
Movement during surgery & $0(0)$ & $0(0)$ \\
$\quad$ Supplemental anesthetics $\quad$ ketamine) & & $0(0)$ \\
Assisted ventilation & & \\
intraoperatively & & $0(0)$ \\
Inotropics or vasoconstrictors & $0(0)$ & \\
$\quad$ during surgery & &
\end{tabular}

Values are presented as mean $\pm \mathrm{SD}$, number (\%), or frequency (\%). Group LS: low-dose (1.0\%) end-tidal sevoflurane concentration group, Group HS: high-dose (2.5\%) end-tidal sevoflurane concentration group. 
at 30 min after arrival in the PACU, it was statistically significant $(\mathrm{P}=0.001)$ (Table 2). The result of the comparison of the PAED scale scores between Group LS and Group HS at 5 min after arrival in the PACU was not statistically significant (P = 0.022); however, at $30 \mathrm{~min}$ after arrival in the PACU, it was statistically significant $(P=0.002)$ (Table 3$)$. Regarding the primary outcome, the difference in the incidence rate of EA between Group LS and Group HS on the basis of a four-point agitation scale score $\geq 3$ at $30 \mathrm{~min}$ after arrival in the PACU was statistically significant (rate difference, $-50.0 \%$ [95\% CI: -71.91 to $-28.09 \%$ ], $\mathrm{P}<0.001)$. Moreover, the difference in the incidence rate of EA between Group LS and Group HS on the basis of a PAED scale score $\geq 10$ at 30 min after arrival in the PACU was statistically significant (rate difference, $-40.0 \%[95 \%$

Table 2. Distribution of Patients according to the Four-point Agitation Scale Scores at Two Time Points

\begin{tabular}{cccc}
\hline $\begin{array}{c}\text { Arrival } \\
\text { in the PACU }\end{array}$ & $\begin{array}{c}\text { Group LS } \\
(\mathrm{n}=20)\end{array}$ & $\begin{array}{c}\text { Group HS } \\
(\mathrm{n}=20)\end{array}$ & P value \\
\hline At 5 min & & & 0.245 \\
1 & $5(25)$ & $7(35)$ & \\
2 & $13(65)$ & $8(40)$ & \\
3 & $2(10)$ & $5(25)$ & \\
4 & $0(0)$ & $0(0)$ & $0.001^{*}$ \\
At 30 min & & & \\
1 & $5(25)$ & $0(0)$ & \\
2 & $15(75)$ & $9(45)$ & \\
3 & $0(0)$ & $9(45)$ & \\
4 & $0(0)$ & $2(10)$ & \\
\hline
\end{tabular}

Values are presented as frequency (\%). PACU: post-anesthetic care unit, Group LS: low-dose (1.0\%) end-tidal sevoflurane concentration group, Group HS: high-dose (2.5\%) end-tidal sevoflurane concentration group. *Statistically significant with a $\mathrm{P}$ value $<0.01$.
CI: -61.47 to $-18.52 \%], \mathrm{P}<0.001)$. However, no difference was observed in the incidence rate of EA at $5 \mathrm{~min}$ after arrival in the PACU; on the four-point agitation scale and PAED scale (Table 4). Among the hemodynamic parameters, the HR and MBP were not statistically different between the two groups ( $\mathrm{P}$ $=0.970$ and $\mathrm{P}=0.627$, respectively) (Figs. 2 and 3 ).

\section{DISCUSSION}

This study showed that the incidence of EA was lower in Group LS than in Group HS, and the combination of caudal block and sevoflurane sedation via a face mask was presumably adequate for performing inguinal hernia repair in children. It prevented inadvertent movement and helped main-

Table 3. Distribution of Patients according to the Pediatric Anesthesia Emergence Delirium Scores at Two Time Points

\begin{tabular}{cccc}
\hline $\begin{array}{c}\text { Arrival } \\
\text { in the PACU }\end{array}$ & $\begin{array}{c}\text { Group LS } \\
(\mathrm{n}=20)\end{array}$ & $\begin{array}{c}\text { Group HS } \\
(\mathrm{n}=20)\end{array}$ & P value \\
\hline At 5 min & & & 0.022 \\
$0-5$ & $6(30)$ & $11(55)$ & \\
$6-9$ & $14(70)$ & $6(30)$ & \\
$10-15$ & $0(0)$ & $3(15)$ & \\
$>15$ & $0(0)$ & $0(0)$ & $0.002 *$ \\
At 30 min & & & \\
$0-5$ & $9(45)$ & $2(10)$ & \\
$6-9$ & $11(55)$ & $8(40)$ & \\
$10-15$ & $0(0)$ & $8(40)$ & \\
$>15$ & $0(0)$ & $2(10)$ & \\
\hline
\end{tabular}

Values are presented as frequency (\%). PACU: post-anesthetic care unit, Group LS: low-dose (1.0\%) end-tidal sevoflurane concentration group, Group HS: high-dose (2.5\%) end-tidal sevoflurane concentration group. * Statistically significant with a $\mathrm{P}$ value $<0.01$.

Table 4. Agitation Incidence Rate at Two Time Points during Observation in the Post-anesthetic Care Unit

\begin{tabular}{lccc}
\hline Variables & Group LS $(\mathrm{n}=20)$ & Group HS $(\mathrm{n}=20)$ & Rate difference, \% $(95 \% \mathrm{Cl})$ \\
\hline $\begin{array}{l}\text { FPAS }(\geq 3) \\
\text { At } 5 \text { min } \\
\text { In the PACU }\end{array}$ & $2(10)$ & $5(25)$ & $-15.0(-38.09$ to 8.087$)$ \\
$\begin{array}{l}\text { At } 30 \text { min } \\
\text { In the PACU }\end{array}$ & $0(0)$ & $10(50)$ & $-50.0(-71.91$ to -28.09$)$ \\
$\begin{array}{l}\text { PAEDS }(\geq 10) \\
\text { At } 5 \text { min } \\
\text { In the PACU } \\
\text { At } 30 \text { min } \\
\text { In the PACU }\end{array}$ & $0(0)$ & $3(15)$ & $-15.0(-30.65$ to 0.65$)$ \\
\hline
\end{tabular}

Values are presented as frequency (\%). Group LS: low-dose (1.0\%) end-tidal sevoflurane concentration group, Group HS: high-dose (2.5\%) end-tidal sevoflurane concentration group, $\mathrm{Cl}$ : confidence interval, FPAS: four-point agitation scale, PAEDS: Pediatric Anesthesia Emergence Delirium scale. *Statistically significant with a P value $<0.01$. 


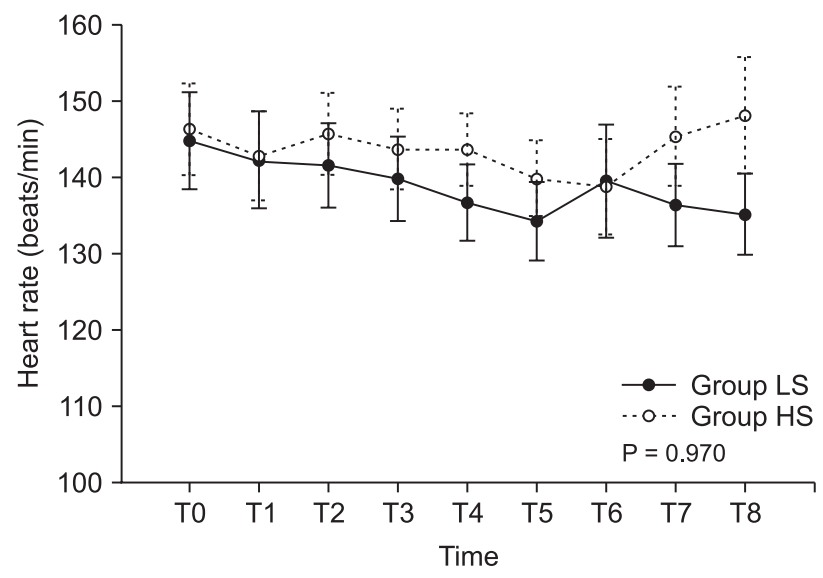

Fig. 2. Heart rate changes. Values are expressed as mean \pm SEM. Group LS: low-dose (1.0\%) end-tidal sevoflurane concentration group, Group HS: high-dose (2.5\%) end-tidal sevoflurane concentration group, TO: arrival at the operation room, T1: immediately before caudal block, T2: immediately after caudal block, T3: 5 min after returning to the supine position, T4: 15 min after returning to the supine position, T5: 5 min before the end of surgery, T6, T7, and T8: 5, 15, and $30 \mathrm{~min}$, respectively, after arrival in the post-anesthetic care unit.

tain a stable hemodynamic condition during surgery.

Caudal block has been established as a practical and safe technique, with low complication rates in sub-umbilical surgery on pediatric patients $[5,10]$. Generally, caudal block is performed as an adjuvant technique during general anesthesia to provide postoperative analgesia. However, intravenous or inhalational sedation with caudal anesthesia has been shown to be a feasible alternative to general anesthesia in pediatric patients undergoing inguinal hernia repair [2-4]. Sedation with caudal block can alleviate the need for endotracheal intubation and may avoid subsequent postoperative complications such as sore throat, hoarseness, and dysphasia $[11,12]$. In neonates and infants in particular, this technique may be more effective than general anesthesia for maintaining spontaneous breathing and attenuating the risk of postoperative apnea and hypoxemia [13]. If sedation with caudal block is used, the dose of intravenous or inhalational anesthetics may be decreased, and this may be beneficial in avoiding the anesthesia-induced effects on neurodevelopment. Kim et al. [14] showed that sevoflurane requirements were lower with caudal block than with general anesthesia alone in children undergoing lower limb surgery. In a study by Weldon et al., the minimum alveolar concentration (MAC) of inhaled anesthetics was reduced by $50 \%$ during the intraoperative phase, after the administration of caudal block [15].

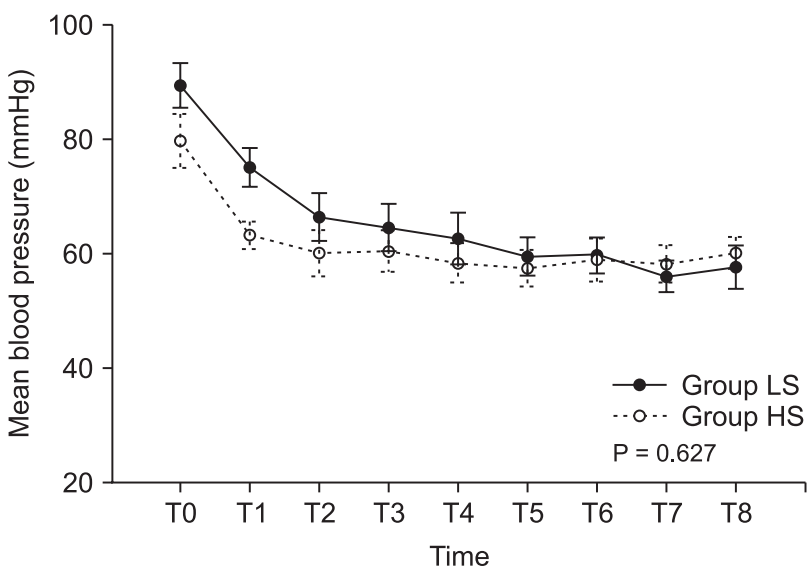

Fig. 3. Mean blood pressure changes. Values are expressed as mean \pm SEM. Group LS: low-dose (1.0\%) end-tidal sevoflurane concentration group, Group HS: high-dose (2.5\%) end-tidal sevoflurane concentration group, TO: arrival at the operation room, T1: immediately before caudal block, T2: immediately after caudal block, T3: 5 min after returning to the supine position, T4: 15 min after returning to the supine position, T5: 5 min before the end of surgery, T6, T7, and T8: 5, 15, and $30 \mathrm{~min}$, respectively, after arrival in the post-anesthetic care unit.

In our institution, sevoflurane sedation with caudal block has been the standard practice of anesthesia for inguinal hernia repair and orchiopexy in pediatric patients. Various sedation protocols can be used; however, ideal sedative drugs for pediatric patients should be easy to administer, provide predictable and reliable sedation, and be associated with rapid recovery and minimal side effects [16]. Inhalation anesthetic masks are commonly used in pediatric anesthesia. Inhalational sevoflurane anesthesia, in particular, has been increasingly used because it has a rapid onset, enables patients to recover quickly, and causes less airway irritation than do the alternatives [17]. However, sevoflurane has undesirable effects such as EA, which occurs in up to $80 \%$ of preschool children [7]. The pathophysiology of EA is unclear, but low patient age and use of volatile anesthetics with low solubility are major contributing factors $[18,19]$. Therefore, if sevoflurane is to be used for pediatric anesthesia, the incidence of EA has to be reduced to avoid self-injury to the patients and disruption of the operative or recovery settings. Although various approaches, including the administration of agents such as opioids, ketamine, and midazolam, have been studied to achieve uneventful emergence [20-22], we intended to determine the suitable concentration of face-mask-delivered sevoflurane that will reduce the incidence of EA in caudal block procedures. 
In this study, we used two agitation rating systems; fourpoint agitation scale and PAED scale. Among the various analysis tools related to EA, PAED scale is a widely used method, but eye contact item of the scale may be affected by the ophthalmic nature of surgery. Therefore, we additionally used a four-point agitation scale to measure EA. The incidence of EA using two analysis tools was higher in Group HS than in Group LS. The rate of EA in Group HS was comparable to that reported in a previous study [7], but the rate in Group LS was much lower. Both sedation concentrations of sevoflurane with caudal block provided adequate surgical conditions for inguinal repair surgery, thus ensuring uneventful completion of the surgery, without any cardiovascular complications, supplemental sedative requirements, and excessive movements during the surgery.

In addition to low patient age and use of sevoflurane, preoperative anxiety is a contributing factor to EA. In this study, we did not assess the degree of preoperative anxiety. However, we administered ketamine and midazolam during preoperative preparation for sedation and relief in patients undergoing caudal block. Therefore, the influence of preoperative anxiety on EA may be somewhat limited in this study.

This study has some limitations. First, although the HR, MBP, and movement were used for estimating the depth of anesthesia, we did not monitor the anesthetic depth by using the bispectral index (BIS) because intraoperative spontaneous ventilation might have caused variations in the BIS. Kern et al. [23] reported wide variations in the BIS during the intraoperative sedation phase $(2.5 \%$ end-tidal sevoflurane through a face mask with peripheral nerve block). Nevertheless, further studies including BIS monitoring are needed for reducing the possibility of inadvertent awareness or suboptimal sedation. Second, we did not record the end-tidal sevoflurane concentration, even though we continuously checked and adjusted the end-tidal sevoflurane concentration during the surgery. It was assumed that if the face mask was correctly applied, the end-tidal sevoflurane concentration might be maintained in a steady-state condition. In the study by Kern et al. [23], the variability in the end-tidal sevoflurane concentration inhaled through the face mask was low, and this was in contrast to the wide variation in the BIS during spontaneous ventilation. However, the possibility of an increased variation in the dosage of inhaled sevoflurane during airway management using the face mask cannot be excluded. Third, high-dose sevoflurane (2.5\%) might be considered an anesthetic dose rather than a sedation dose, even though we selected this dose on the basis of the findings of another study. Fourth [23], we did not consider the value of adjusting the end-tidal sevoflurane concentration. Age affects the MAC of sevoflurane to different degrees. Future studies should consider the importance of adjusting the endtidal sevoflurane concentration. Fifth, we used ketamine and midazolam as preoperative sedatives, as part of the standard care at our institution. Ketamine and midazolam have been shown to decrease the incidence of EA in some studies [24,25], but other studies have reported conflicting findings [26]. Both drugs might be responsible for EA; nevertheless, performing awake caudal anesthesia in the pediatric population is unethical. Moreover, since neither drug biased the results, they were administered to all children. Finally, although EA scoring in the PACU was double-blinded, this study was not double-blinded intraoperatively. This might affect the credibility of the observed findings.

In conclusion, sevoflurane face-mask sedation with caudal block may be considered an alternative anesthetic technique in children undergoing inguinal hernia repair, and $1.0 \%$ sevoflurane may be more effective than $2.5 \%$ sevoflurane in preventing EA.

\section{CONFLICTS OF INTEREST}

No potential conflict of interest relevant to this article was reported.

\section{ORCID}

Eun Kyung Choi: https://orcid.org/0000-0001-5758-6741

Suyong Park: https://orcid.org/0000-0002-2735-4760

Ki-bum Park: https://orcid.org/0000-0002-1432-8301

Kyung Hwa Kwak: https://orcid.org/0000-0003-0819-1764

\section{REFERENCES}

1. Veyckemans F, Van Obbergh LJ, Gouverneur JM. Lessons from 1100 pediatric caudal blocks in a teaching hospital. Reg Anesth 1992; 17: 119-25.

2. Bong CL, Yeo AS, Fabila T, Tan JS. A pilot study of dexmedetomidine sedation and caudal anesthesia for inguinal hernia repair in 
infants. Paediatr Anaesth 2016; 26: 621-7.

3. Balent E, Edwards M, Lustik M, Martin P. Caudal anesthesia with sedation for inguinal hernia repair in high risk neonates. J Pediatr Surg 2014; 49: 1304-7.

4. Choi EK, Ro Y, Park SS, Park KB. The use of EMLA cream reduces the pain of skin puncture associated with caudal block in children. Korean J Anesthesiol 2016; 69: 149-54.

5. Gunter J. Caudal anesthesia in children: a survey. Anesthesiology 1991; 75: A936.

6. Sikich N, Lerman J. Development and psychometric evaluation of the pediatric anesthesia emergence delirium scale. Anesthesiology 2004; 100: 1138-45.

7. Voepel-Lewis T, Malviya S, Tait AR. A prospective cohort study of emergence agitation in the pediatric postanesthesia care unit. Anesth Analg 2003; 96: 1625-30.

8. Finkel JC, Cohen IT, Hannallah RS, Patel KM, Kim MS, Hummer KA, et al. The effect of intranasal fentanyl on the emergence characteristics after sevoflurane anesthesia in children undergoing surgery for bilateral myringotomy tube placement. Anesth Analg 2001; 92: 1164-8.

9. Son JS, Jang E, Oh MW, Lee JH, Han YJ, Ko S. Erratum: a comparison of postoperative emergence agitation between sevoflurane and thiopental anesthesia induction in pediatric patients. Korean J Anesthesiol 2016; 69: 100. Erratum for: Korean J Anesthesiol 2015; 68: 373-8.

10. Giaufré E, Dalens B, Gombert A. Epidemiology and morbidity of regional anesthesia in children: a one-year prospective survey of the French-Language Society of Pediatric Anesthesiologists. Anesth Analg 1996; 83: 904-12.

11. Combes X, Schauvliege F, Peyrouset O, Motamed C, Kirov K, Dhonneur $\mathrm{G}$, et al. Intracuff pressure and tracheal morbidity: influence of filling with saline during nitrous oxide anesthesia. Anesthesiology 2001; 95: 1120-4.

12. Christensen AM, Willemoes-Larsen H, Lundby L, Jakobsen KB. Postoperative throat complaints after tracheal intubation. Br J Anaesth 1994; 73: 786-7.

13. Sims C, Johnson CM. Postoperative apnoea in infants. Anaesth Intensive Care 1994; 22: 40-5.

14. Kim SH, Chun DH, Chang CH, Kim TW, Kim YM, Shin YS. Effect of caudal block on sevoflurane requirement for lower limb surgery in children with cerebral palsy. Paediatr Anaesth 2011; 21: 394-8.

15. Weldon BC, Bell M, Craddock T. The effect of caudal analgesia on emergence agitation in children after sevoflurane versus halothane anesthesia. Anesth Analg 2004; 98: 321-6.
16. Mallory MD, Baxter AL, Kost SI; Pediatric Sedation Research Consortium. Propofol vs pentobarbital for sedation of children undergoing magnetic resonance imaging: results from the Pediatric Sedation Research Consortium. Paediatr Anaesth 2009; 19: 601-11.

17. Lerman J, Davis PJ, Welborn LG, Orr RJ, Rabb M, Carpenter R, et al. Induction, recovery, and safety characteristics of sevoflurane in children undergoing ambulatory surgery. A comparison with halothane. Anesthesiology 1996; 84: 1332-40.

18. Aono J, Ueda W, Mamiya K, Takimoto E, Manabe M. Greater incidence of delirium during recovery from sevoflurane anesthesia in preschool boys. Anesthesiology 1997; 87: 1298-300.

19. Cravero J, Surgenor S, Whalen K. Emergence agitation in paediatric patients after sevoflurane anaesthesia and no surgery: a comparison with halothane. Paediatr Anaesth 2000; 10: 419-24.

20. Bortone L, Bertolizio G, Engelhardt T, Frawley G, Somaini M, Ingelmo PM. The effect of fentanyl and clonidine on early postoperative negative behavior in children: a double-blind placebo controlled trial. Paediatr Anaesth 2014; 24: 614-9.

21. Cho EJ, Yoon SZ, Cho JE, Lee HW. Comparison of the effects of 0.03 and $0.05 \mathrm{mg} / \mathrm{kg}$ midazolam with placebo on prevention of emergence agitation in children having strabismus surgery. Anesthesiology 2014; 120: 1354-61.

22. Abu-Shahwan I, Chowdary K. Ketamine is effective in decreasing the incidence of emergence agitation in children undergoing dental repair under sevoflurane general anesthesia. Paediatr Anaesth 2007; 17: 846-50.

23. Kern D, Fourcade O, Mazoit JX, Minville V, Chassery C, Chausseray G, et al. The relationship between bispectral index and endtidal concentration of sevoflurane during anesthesia and recovery in spontaneously ventilating children. Paediatr Anaesth 2007; 17: 249-54.

24. Kim KM, Lee KH, Kim YH, Ko MJ, Jung JW, Kang E. Comparison of effects of intravenous midazolam and ketamine on emergence agitation in children: randomized controlled trial. J Int Med Res 2016; 44: 258-66.

25. Ko YP, Huang CJ, Hung YC, Su NY, Tsai PS, Chen CC, et al. Premedication with low-dose oral midazolam reduces the incidence and severity of emergence agitation in pediatric patients following sevoflurane anesthesia. Acta Anaesthesiol Sin 2001; 39: 169-77.

26. Breschan C, Platzer M, Jost R, Stettner H, Likar R. Midazolam does not reduce emergence delirium after sevoflurane anesthesia in children. Paediatr Anaesth 2007; 17: 347-52. 